\title{
Component splitting for semi-discrete Maxwell equations
}

\author{
J.G. Verwer
}

Received: 23 March 2010 / Accepted: 2 November 2010 / Published online: 17 November 2010

(C) Springer Science + Business Media B.V. 2010

\begin{abstract}
A time-integration scheme for semi-discrete linear Maxwell equations is proposed. Special for this scheme is that it employs component splitting. The idea of component splitting is to advance the greater part of the components of the semidiscrete system explicitly in time and the remaining part implicitly. The aim is to avoid severe step size restrictions caused by grid-induced stiffness emanating from locally refined space grids. The proposed scheme is a blend of an existing secondorder composition scheme which treats wave terms explicitly and the second-order implicit trapezoidal rule. The new blended scheme retains the composition property enabling higher-order composition.
\end{abstract}

Keywords Numerical integration · Component splitting · Wave equations · Maxwell equations

Mathematics Subject Classification (2000) 65L05 · 65L20 · 65M12 - 65M20

\section{Introduction}

In this paper we study the numerical integration of linear ODE systems

$$
\left(\begin{array}{cc}
M_{u} & 0 \\
0 & M_{v}
\end{array}\right)\left(\begin{array}{l}
u^{\prime} \\
v^{\prime}
\end{array}\right)=\left(\begin{array}{cc}
0 & -K \\
K^{T} & -D
\end{array}\right)\left(\begin{array}{l}
u \\
v
\end{array}\right)+\left(\begin{array}{l}
f^{u}(t) \\
f^{v}(t)
\end{array}\right) .
$$

Communicated by Per Lötstedt.

Jan Verwer passed away on 16 February 2011, while this paper was in press.

J.G. Verwer $(\bowtie)$

Centrum Wiskunde \& Informatica (CWI), Science Park 123, 1098 XG Amsterdam, The Netherlands

e-mail: Jan.Verwer@cwi.nl 
This partitioned form is generic for semi-discrete linear first-order wave equations. In particular, it covers common spatial discretizations of the Maxwell equations

$$
\begin{aligned}
\mu \partial_{t} H & =-\nabla \times E, \\
\varepsilon \partial_{t} E & =\nabla \times H-\sigma E-J_{E},
\end{aligned}
$$

including the classical staggered finite-difference and various finite-element discretizations. The similarity between (1.1) and (1.2) shall be clear. The vectors $u=u(t)$ and $v=v(t)$ are the unknown vector (grid) functions approximating the values of the magnetic field $H$ and electric field $E$, respectively. The matrices $K$ and $K^{T}$ emanate from the curl operator $\nabla \times$. In particular, we will assume throughout this paper that element wise

$$
K \sim \frac{1}{h}, \quad h \rightarrow 0,
$$

where $h$ parameterizes the distance of the (possibly nonuniform) space grid and the dimensions of $K$ and $u$ and $v$. The matrix $D$ is associated with the dissipative conduction term $-\sigma E$ and the matrices $M_{u}, M_{v}$ typically represent mass matrices arising with finite elements. The functions $f^{u}(t)$ and $f^{v}(t)$ are source terms. Normally $f^{v}$ represents the given source current $J_{E}$, but $f^{u}$ and $f^{v}$ may also contain boundary data.

We could have also started from

$$
\left(\begin{array}{l}
u^{\prime} \\
v^{\prime}
\end{array}\right)=\left(\begin{array}{cc}
0 & -K \\
K^{T} & -D
\end{array}\right)\left(\begin{array}{l}
u \\
v
\end{array}\right)+\left(\begin{array}{l}
f^{u}(t) \\
f^{v}(t)
\end{array}\right),
$$

because our integration schemes can be implemented for either choice. In particular, results for this somewhat more simple formulation always carry over to (1.1) and vice versa, see e.g. [1]. For convenience of notation and presentation, we will therefore proceed with (1.4). Herein the damping matrix $D$ may be assumed symmetric, non-negative definite. For zero $D$ the matrix of (1.4) is skew-symmetric. As a consequence, when omitting the source terms $f^{u}$ and $f^{v}$, the exact solution satisfies

$$
\frac{d}{d t}\left(\|u(t)\|^{2}+\|v(t)\|^{2}\right)=-2\langle D v, v\rangle \leq 0,
$$

where $\langle\cdot, \cdot\rangle$ is the $l_{2}$ inner product (the standard scalar product) and $\|\cdot\|$ the corresponding norm. So, without damping we have norm preservation (energy conservation) and with damping norm dissipation. In both cases we speak of stability. An appropriate numerical method should mimic these solution norm properties sufficiently accurately.

The contribution of this paper is a numerical time-integration scheme for semidiscrete, linear Maxwell equations (1.4) that is designed to enable component splitting. The idea of component splitting is to advance part of the components of the semi-discrete system explicitly in time and the remaining part implicitly. The aim is to overcome severe stability step size restrictions in cases of grid-induced stiffness emanating from locally refined space grids. For example, unstructured finite-element grids on complex domains may contain elements much smaller than found elsewhere 
just to accommodate a complicated spatial geometry [3]. If the number of these very small elements amounts to only a relatively small fraction of the total number of elements, we speak of grid-induced stiffness, because this requires standard explicit schemes to take small step sizes limited by the very small elements only but to be used for all elements of the grid including coarser ones.

In such cases a component splitting scheme may prove very useful as it overcomes the stability step size limitation at the expense of solving per time step a small sized symmetric positive definite system of linear algebraic equations. The component splitting technique we propose in this paper is akin to the component splitting in our recent preprint [13] on the two-step Crank-Nicolson-Leapfrog scheme. The new scheme is a blend of a well-known second-order, explicit (in the wave terms) composition scheme and the second-order implicit trapezoidal rule. Thus the new blended scheme is one-step and retains the composition property enabling higher-order composition. The new scheme also bears a relationship to a component splitting scheme discussed in [3] which is especially designed for a discontinuous Galerkin discretization. A novel local time-stepping technique for second-order wave equations discretized in space by a continuous or discontinuous finite element method has been proposed in [2] and is further discussed in [4]. This local time-stepping technique also serves to overcome step size limitations by grid-induced stiffness.

In Sect. 2 we will briefly review the second-order composition scheme and the trapezoidal rule. Section 3 is devoted to our component splitting scheme. Here we also discuss conservation and convergence properties. In particular, we will prove that our new scheme retains its second order in time for spatial grid size $h \rightarrow 0$. Thus the scheme does not suffer from order reduction which is not a priori clear with splitting. Numerical results are presented in Sect. 4. The paper concludes with Sect. 5 on some plans for future work. Also a few remarks on the approach from [2] and [4] are included in this final section.

\section{The composition scheme and the trapezoidal rule}

The composition scheme is given by

$$
\begin{aligned}
\frac{u_{n+1 / 2}-u_{n}}{\tau} & =-\frac{1}{2} K v_{n}+\frac{1}{2} f^{u}\left(t_{n}\right), \\
\frac{v_{n+1}-v_{n}}{\tau} & =K^{T} u_{n+1 / 2}-\frac{1}{2} D\left(v_{n}+v_{n+1}\right)+\frac{1}{2}\left(f^{v}\left(t_{n}\right)+f^{v}\left(t_{n+1}\right)\right), \\
\frac{u_{n+1}-u_{n+1 / 2}}{\tau} & =-\frac{1}{2} K v_{n+1}+\frac{1}{2} f^{u}\left(t_{n+1}\right) .
\end{aligned}
$$

This one-step method steps from $\left(u_{n}, v_{n}\right)$ to $\left(u_{n+1}, v_{n+1}\right)$ with step size $\tau$. Here $u_{n}$ denotes the approximation to the exact solution $u\left(t_{n}\right)$, etc., and $\tau=t_{n+1}-t_{n}$. The method is explicit in the wave terms and implicit in $D$ (the trapezoidal rule). If $D$ is block-diagonal with a small bandwidth, this implicitness comes with little costs. For $n \geq 1$ the third-stage derivative computation can be copied to the first stage at the next time step to save computational work. Per time step this method thus is very 
economical as it actually requires a single righthand side evaluation per time step (for zero $D$ ), while it is second-order consistent (this follows immediately from its symmetry). Assuming the source function evaluations to be cheap, the costs per time step amount mainly to one matrix-vector multiplication with $K$ and one with $K^{T}$.

This composition scheme is well-known, in particular for zero $D$, in the literature on geometric integration [5]. With regard to time stepping it bears a close resemblance to the popular Yee-scheme [14] from electromagnetism and to Verlet's method from molecular dynamics [11]. For the Maxwell equations it has for example been studied in [9] and [1].

A step size bound for stability for the general system (1.4) is not known, ${ }^{1}$ except when the diagonal matrix $D$ is given by the unit matrix times a constant non-negative scalar, say $\alpha$. Then we have stability in the inner product norm if [1]

$$
\tau s_{\max }<2 \text { if } \alpha=0 \text { and } \tau s_{\max } \leqslant 2 \text { if } \alpha>0 \text {, }
$$

where $s_{\max }$ is the maximal square root of the eigenvalues of $K^{T} K$. Because these eigenvalues are proportional to $h^{-2}$, for time stepping stability a relation $\tau \sim h$ for $h \rightarrow 0$ is required. This result applies to (1.2) with $\alpha=\sigma / \varepsilon$ if $\varepsilon$ and $\sigma$ are constant scalars. Note that the conduction puts no limit on $\tau$ and that the requirement $\tau \sim h$ for $h \rightarrow 0$ is common for explicit methods. On the other hand, on strongly non-uniform grids with locally very small cells, the step size limitation for the wave terms can be truly restrictive.

For more details and results on (2.1) we refer to [1]. Assuming (1.3), one of the results states that if $\tau \sim h$ its second ODE order is maintained for $h \rightarrow 0$ (no order reduction with stiff source terms). Another result is that, with zero source terms,

$$
\begin{aligned}
& \frac{\left(\left\|u_{n+1}\right\|^{2}+\left\|v_{n+1}\right\|^{2}-\left(\left\|u_{n}\right\|^{2}+\left\|v_{n}\right\|^{2}\right)\right.}{\tau} \\
& =-2\left\langle D\left(\frac{v_{n}+v_{n+1}}{2}\right), \frac{v_{n}+v_{n+1}}{2}\right\rangle \\
& \quad-\frac{1}{4} \tau\left(\left\langle K v_{n}, K v_{n}\right\rangle-\left\langle K v_{n+1}, K v_{n+1}\right\rangle\right) .
\end{aligned}
$$

It thus follows that with a zero damping term and zero source terms, we have (energy) conservation if and only if $\left\langle K v_{n}, K v_{n}\right\rangle=\left\langle K v_{n+1}, K v_{n+1}\right\rangle$, cf. (1.5). In general this will not hold. What is conserved, however, is the $\mathcal{O}\left(\tau^{2}\right)$-perturbed quantity

$$
\left\|u_{n}\right\|^{2}+\left\|v_{n}\right\|^{2}-\frac{1}{4} \tau^{2}\left\langle K v_{n}, K v_{n}\right\rangle
$$

showing that the conservation behavior is actually very good. Herewith it is of course tacitly assumed that $\tau$ is limited such that the method integrates in a stable way, something which cannot be concluded from this result due to the minus sign in front of the third term.

\footnotetext{
${ }^{1}$ We mean a bound expressed in properties of $K, K^{T}$ and $D$ only.
} 
The component splitting scheme discussed in Sect. 3 is a blend of (2.1) and the 2nd-order, unconditionally stable implicit trapezoidal (Crank-Nicolson) rule. Like (2.1) we write it in the three-stage form

$$
\begin{aligned}
\frac{u_{n+1 / 2}-u_{n}}{\tau}= & -\frac{1}{2} K v_{n}+\frac{1}{2} f^{u}\left(t_{n}\right), \\
\frac{v_{n+1}-v_{n}}{\tau}= & \frac{1}{2} K^{T}\left(u_{n}+u_{n+1}\right)-\frac{1}{2} D\left(v_{n}+v_{n+1}\right) \\
& +\frac{1}{2}\left(f^{v}\left(t_{n}\right)+f^{v}\left(t_{n+1}\right)\right), \\
\frac{u_{n+1}-u_{n+1 / 2}}{\tau}= & -\frac{1}{2} K v_{n+1}+\frac{1}{2} f^{u}\left(t_{n+1}\right) .
\end{aligned}
$$

For zero sources this scheme mimics (1.5) through

$$
\begin{aligned}
& \frac{\left(\left\|u_{n+1}\right\|^{2}+\left\|v_{n+1}\right\|^{2}\right)-\left(\left\|u_{n}\right\|^{2}+\left\|v_{n}\right\|^{2}\right)}{\tau} \\
& =-2\left\langle D \frac{v_{n+1}+v_{n}}{2}, \frac{v_{n+1}+v_{n}}{2}\right\rangle, \quad \forall \tau>0,
\end{aligned}
$$

revealing unconditional stability and energy conservation. On strongly non-uniform grids with locally very small cells unconditional stability seems attractive. However, our experience from [12] indicates that even with locally very small cells the overhead for implicit solves can become too large to render the trapezoidal rule competitive to (2.1). With component splitting we seek to reduce this overhead by treating only a small portion of the components implicitly.

\section{The component splitting scheme}

The schemes (2.1) and (2.5) differ only in the second stage for vector $v$. This suggests to blend the two into

$$
\begin{aligned}
\frac{u_{n+1 / 2}-u_{n}}{\tau}= & -\frac{1}{2} K v_{n}+\frac{1}{2} f^{u}\left(t_{n}\right), \\
\frac{v_{n+1}-v_{n}}{\tau}= & K_{0}^{T} u_{n+1 / 2}+\frac{1}{2} K_{1}^{T}\left(u_{n}+u_{n+1}\right) \\
& -\frac{1}{2} D\left(v_{n}+v_{n+1}\right)+\frac{1}{2}\left(f^{v}\left(t_{n}\right)+f^{v}\left(t_{n+1}\right)\right), \\
\frac{u_{n+1}-u_{n+1 / 2}}{\tau}= & -\frac{1}{2} K v_{n+1}+\frac{1}{2} f^{u}\left(t_{n+1}\right),
\end{aligned}
$$

where

$$
K=K_{0}+K_{1}
$$


is a matrix splitting that is still to be defined. By this splitting the method is explicit for $K_{0}$ and implicit for $K_{1}$. For zero $K_{1}$ we recover (2.1) and for zero $K_{0}$ method (2.5). Note that matrix $D$ and the source terms are not split and that for $n \geq 1$ the computation of the third stage can again be reused at the first stage of the next time step, which enhances efficiency. Because the scheme is also symmetric, it is 2ndorder consistent for any given semi-discrete Maxwell system (1.4) of fixed dimension. We will prove below that like its constituent schemes (2.1) and (2.5), the component splitting scheme (3.1) retains its second ODE order under stable simultaneous spacetime grid refinement.

The splitting (3.2) is very general. Our aim, however, is to adopt the idea of component splitting proposed in [13] to restrict the implicit computation of $u_{n+1}, v_{n+1}$ only to those components which impede implicitness for stability reasons. Let us therefore first describe how the implicit computation is done. From the third stage of (3.1) follows

$$
K_{1}^{T} u_{n+1}=-\frac{1}{2} \tau K_{1}^{T} K v_{n+1}+K_{1}^{T}\left(u_{n+1 / 2}+\frac{1}{2} \tau f^{u}\left(t_{n+1}\right)\right) .
$$

Substitution into the second stage then defines $v_{n+1}$ by

$$
\mathcal{M} v_{n+1}=b_{n+1}
$$

where

$$
\begin{aligned}
\mathcal{M}= & I+\frac{1}{4} \tau^{2} K_{1}^{T} K+\frac{1}{2} \tau D \\
b_{n+1}= & v_{n}+\tau K_{0}^{T} u_{n+1 / 2}+\frac{1}{2} \tau K_{1}^{T}\left(u_{n}+u_{n+1 / 2}+\frac{1}{2} \tau f^{u}\left(t_{n+1}\right)\right) \\
& -\frac{1}{2} \tau D v_{n}+\frac{1}{2} \tau\left(f^{v}\left(t_{n}\right)+f^{v}\left(t_{n+1}\right)\right) .
\end{aligned}
$$

Hence we can solve $v_{n+1}$ from the linear equation (3.4) and successively $u_{n+1}$ directly from the third stage formula. For $K_{1}=K$, recovering the implicit trapezoidal rule, matrix $\mathcal{M}-\frac{1}{2} \tau D$ is symmetric positive definite, since for any useful spatial discretization $K^{T} K$ is symmetric semi-positive definite. With the splitting we wish to also have $K_{1}^{T} K$ symmetric (and hopefully semi-positive definite), since this facilitates the solution of (3.4), either directly or iteratively.

To this end we define $K_{1}$ as follows:

$$
K_{1}=S_{u} K
$$

where $S_{u}$ is a diagonal matrix of dimension the length of $u$ and with entries $S_{u}^{(j j)}$ obeying the rule

$$
S_{u}^{(j j)}= \begin{cases}0, & \text { component } u_{j} \text { of } u \text { to be treated explicitly, } \\ 1, & \text { component } u_{j} \text { of } u \text { to be treated implicitly. }\end{cases}
$$

Again, this definition of $S$ is rather general so that we have some freedom in choosing $S$ in an actual application. With this definition, however, we do have the aimed 
symmetry of $K_{1}^{T} K$, since $K_{1}^{T} K=K^{T} S_{u} K=K^{T} S_{u} S_{u} K=K_{1}^{T} K_{1}$, giving

$$
\mathcal{M}=I+\frac{1}{4} \tau^{2} K_{1}^{T} K_{1}+\frac{1}{2} \tau D
$$

If component splitting works as anticipated, this matrix $\mathcal{M}$ will be significantly more sparse than without splitting, enabling us to solve the linear systems (3.4) at significantly lower costs.

\subsection{Conservation}

Remove the sources in (3.1) and substitute half of $u_{n+1 / 2}$ from the first stage and half of $u_{n+1 / 2}$ from the third one into the second stage. Together with the final expression for $u_{n+1}$ this yields

$$
\begin{aligned}
u_{n+1}-u_{n}= & -\frac{1}{2} \tau K\left(v_{n}+v_{n+1}\right), \\
v_{n+1}-v_{n}= & \tau K_{0}^{T}\left(\frac{1}{2} u_{n}+\frac{1}{2} u_{n+1}-\frac{1}{4} \tau K v_{n}+\frac{1}{4} \tau K v_{n+1}\right) \\
& +\frac{1}{2} \tau K_{1}^{T}\left(u_{n}+u_{n+1}\right)-\frac{1}{2} \tau D\left(v_{n}+v_{n+1}\right) .
\end{aligned}
$$

Taking inner products with $u_{n+1}+u_{n}$ and $v_{n+1}+v_{n}$ yields, respectively,

$$
\begin{aligned}
\left\|u_{n+1}\right\|^{2}-\left\|u_{n}\right\|^{2}= & -\frac{1}{2} \tau\left\langle K^{T}\left(u_{n}+u_{n+1}\right), v_{n}+v_{n+1}\right\rangle, \\
\left\|v_{n+1}\right\|^{2}-\left\|v_{n}\right\|^{2}= & \frac{1}{2} \tau\left\langle K_{1}^{T}\left(u_{n}+u_{n+1}\right), v_{n}+v_{n+1}\right\rangle \\
& -\frac{1}{2} \tau\left\langle D\left(v_{n}+v_{n+1}\right), v_{n}+v_{n+1}\right\rangle \\
& +\frac{1}{2} \tau\left\langle K_{0}^{T}\left(u_{n}+u_{n+1}\right), v_{n}+v_{n+1}\right\rangle \\
& +\frac{1}{4} \tau^{2}\left\langle K_{0}^{T} K\left(v_{n+1}-v_{n}, v_{n}+v_{n+1}\right\rangle .\right.
\end{aligned}
$$

Hence,

$$
\begin{aligned}
& \frac{\left(\left\|u_{n+1}\right\|^{2}+\left\|v_{n+1}\right\|^{2}\right)-\left(\left\|u_{n}\right\|^{2}+\left\|v_{n}\right\|^{2}\right)}{\tau} \\
& \quad=\frac{1}{4} \tau\left\langle K_{0}^{T} K\left(v_{n+1}-v_{n}\right), v_{n+1}+v_{n}\right\rangle-2\left\langle D \frac{v_{n}+v_{n+1}}{2}, \frac{v_{n}+v_{n+1}}{2}\right\rangle .
\end{aligned}
$$

Since by definition of $K_{1}=S_{u} K$ the matrix $K_{0}^{T} K$ is symmetric, it follows that for zero damping matrix $D$ we have the conserved quantity

$$
\begin{aligned}
\left\|u_{n}\right\|^{2}+\left\|v_{n}\right\|^{2}-\frac{1}{4} \tau^{2}\left\langle K v_{n}, K_{0} v_{n}\right\rangle= & \left\|u_{n}\right\|^{2}+\left\|v_{n}\right\|^{2}-\frac{1}{4} \tau^{2}\left(\left\langle K v_{n}, K v_{n}\right\rangle\right. \\
& \left.-\left\langle S_{u} K v_{n}, S_{u} K v_{n}\right\rangle\right)
\end{aligned}
$$


For the unit diagonal matrix $S_{u}$ we recover the exact conservation property of the implicit trapezoidal rule and for zero diagonal matrix $S_{u}$ the conservation property (2.4) of the explicit scheme. Since in general the entries of the diagonal matrix $S_{u}$ are either zero or one, with component splitting the deviation from the exact energy is reduced compared to the explicit scheme.

\subsection{Convergence}

This section is central in the paper. For our component splitting scheme (3.1) applied to system (1.3), (1.4) it sketches an error analysis on the following result. Let $u_{h}(t)$ and $v_{h}(t)$ denote the grid functions for the true underlying PDE solution at time $t$ restricted to the used space grid and assume them to be three times continuously differentiable on a given, finite time interval $[0, T]$. For a Lax-Richtmyer stable space-time grid refinement $\tau \sim h, h \rightarrow 0$, the component splitting scheme approximations then converge with temporal order two to $u_{h}, v_{h}$ on $[0, T]$ for any splitting (3.2), (3.6). This means that neither the splitting nor stiff sources do introduce temporal order reduction, something which for splitting methods and stiff source terms is not a priori clear. Further note that the assumptions for convergence are similar as for the trapezoidal rule and the explicit scheme (2.1).

The derivations in the remainder of this section follow a method of lines analysis related to that of [10] and [6, Sect. II.2]. Although these earlier contributions deal with explicit Runge-Kutta methods, the notion on order reduction is the same and the type of derivations is similar. The proof of second temporal order in the PDE sense presented here is organized in three subsections. In Sect. 3.2.1 we will introduce the so-called perturbed scheme obtained by substituting the true PDE solution restricted to the assumed space grid into our component splitting scheme (3.1). Herewith we introduce defects (truncation errors) composed of a temporal and a spatial error part. Our focus lies on temporal order, so for simplicity of derivation we will omit the spatial error part after this subsection. This is not essential. For our purpose the spatial error part may be omitted without loss of generality, cf. [10] and [6, Sect. II.2]. In Sect. 3.2.2 we derive the common temporal recurrence for the full global error: the difference of the PDE solution restricted to the space grid and the numerical solution on this grid generated by scheme (3.1). Here we point out that this global error scheme needs to be transformed to overcome a spatial inconsistency in the local error emanating from component splitting. The crucial observation hereby is that this spatial inconsistency enters the temporal error by the negative power $h^{-1}$ which kills one power of $\tau$ as we assume $\tau \sim h, h \rightarrow 0$ (order reduction). Fortunately, this order reduction is present in the local error only and cancels in the transition from local to global errors. That this cancelation occurs can be proved by transforming the global error scheme, which is shown in the third Sect. 3.2.3.

\subsubsection{The perturbed scheme}

Substitution of $u_{h}$ and $v_{h}$ into (1.4) reveals the spatial truncation errors which we denote by $\sigma_{h}^{u}$ and $\sigma_{h}^{v}$, that is,

$$
\begin{aligned}
& u_{h}^{\prime}(t)=-K v_{h}(t)+f^{u}(t)+\sigma_{h}^{u}(t), \\
& v_{h}^{\prime}(t)=K^{T} u_{h}(t)-D v_{h}(t)+f^{v}(t)+\sigma_{h}^{v}(t) .
\end{aligned}
$$


Next, substitution of the exact solutions $u_{h}(t), v_{h}(t)$ into the component splitting scheme (3.1) gives what we call the perturbed scheme containing defects (truncation errors) composed of a temporal and a spatial error part. Let $\delta_{k}$ denote the defects for the stages $k=1,2,3$ (other sub indices are omitted to simplify notation). We then have as perturbed scheme

$$
\begin{aligned}
\frac{u_{h}\left(t_{n+1 / 2}\right)-u_{h}\left(t_{n}\right)}{\tau}= & -\frac{1}{2} K v_{h}\left(t_{n}\right)+\frac{1}{2} f^{u}\left(t_{n}\right)+\delta_{1}, \\
\frac{v_{h}\left(t_{n+1}\right)-v_{h}\left(t_{n}\right)}{\tau}= & K_{0}^{T} u_{h}\left(t_{n+1 / 2}\right)+\frac{1}{2} K_{1}^{T}\left(u_{h}\left(t_{n}\right)+u_{h}\left(t_{n+1}\right)\right) \\
& -\frac{1}{2} D\left(v_{h}\left(t_{n}\right)+v_{h}\left(t_{n+1}\right)\right) \\
& +\frac{1}{2}\left(f^{v}\left(t_{n}\right)+f^{v}\left(t_{n+1}\right)\right)+\delta_{2}, \\
\frac{u_{h}\left(t_{n+1}\right)-u_{h}\left(t_{n+1 / 2}\right)}{\tau}= & -\frac{1}{2} K v_{h}\left(t_{n+1}\right)+\frac{1}{2} f^{u}\left(t_{n+1}\right)+\delta_{3} .
\end{aligned}
$$

Eliminating all source term $f^{u}, f^{v}$ contributions in the defect expressions through (3.13) yields

$$
\begin{aligned}
\delta_{1}= & \frac{u_{h}\left(t_{n+1 / 2}\right)-u_{h}\left(t_{n}\right)}{\tau}-\frac{1}{2} u_{h}^{\prime}\left(t_{n}\right)+\frac{1}{2} \sigma_{h}^{u}\left(t_{n}\right), \\
\delta_{2}= & \frac{v_{h}\left(t_{n+1}\right)-v_{h}\left(t_{n}\right)}{\tau}-\frac{1}{2}\left(v_{h}^{\prime}\left(t_{n}\right)+v_{h}^{\prime}\left(t_{n+1}\right)\right) \\
& -K_{0}^{T}\left(u_{h}\left(t_{n+1 / 2}\right)-\frac{1}{2}\left(u_{h}\left(t_{n}\right)+u_{h}\left(t_{n+1}\right)\right)\right)+\frac{1}{2}\left(\sigma_{h}^{v}\left(t_{n}\right)+\sigma_{h}^{v}\left(t_{n+1}\right)\right), \\
\delta_{3}= & \frac{u_{h}\left(t_{n+1}\right)-u_{h}\left(t_{n+1 / 2}\right)}{\tau}-\frac{1}{2} u_{h}^{\prime}\left(t_{n+1}\right)+\frac{1}{2} \sigma_{h}^{u}\left(t_{n+1}\right) .
\end{aligned}
$$

Herein we can distinguish the temporal error parts and the spatial error parts contained in the $\sigma_{h}^{u}, \sigma_{h}^{v}$ contributions. As mentioned above, our interest lies in the temporal errors. We therefore simplify our derivations by omitting these spatial contributions. This is not essential. Carrying the spatial contributions along in the derivations just complicates the formulas and will not lead to different conclusions for the temporal errors. Finally, the formal Taylor expansion at $t_{n+1 / 2}$ delivers the temporal defect expressions

$$
\begin{aligned}
& \delta_{1}=\sum_{j=2}\left(\frac{1}{(j-1) !}-\frac{1}{j !}\right) \frac{(-1)^{j}}{2^{j}} \tau^{j-1} u_{h}^{(j)}, \quad \delta_{2}=\delta_{4}+\left(K-K_{1}\right)^{T} \delta_{5}, \\
& \delta_{3}=\sum_{j=2}\left(\frac{1}{j !}-\frac{1}{(j-1) !}\right) \frac{1}{2^{j}} \tau^{j-1} u_{h}^{(j)}, \\
& \delta_{4}=\sum_{j=2^{\prime}} \frac{-j}{2^{j}(j+1) !} \tau^{j} v_{h}^{(j+1)}, \quad \delta_{5}=\sum_{j=2^{\prime}} \frac{1}{2^{j} j !} \tau^{j} u_{h}^{(j)},
\end{aligned}
$$


where $j=2^{\prime}$ means even values for $j$ only, $\delta_{4}$ and $\delta_{5}$ are auxiliary, and $u_{h}^{(j)}$ denotes the $j$-th derivative of $u_{h}(t)$ at $t=t_{n+1 / 2}$, etc. Also note that $\delta_{1}$ and $\delta_{3}$ start of with $\tau$ and $\delta_{4}$ and $\delta_{5}$ with $\tau^{2}$. Further, except for $\delta_{2}$, all defects just contain higher temporal derivatives of the true PDE solution.

\subsubsection{The global error recursion}

Next we introduce the global errors $\varepsilon_{n}^{u}=u_{h}\left(t_{n}\right)-u_{n}$ and $\varepsilon_{n}^{v}=v_{h}\left(t_{n}\right)-v_{n}$ and the intermediate global error $\varepsilon_{n+1 / 2}^{u}=u_{h}\left(t_{n+1 / 2}\right)-u_{n+1 / 2}$. Subtracting (3.1) from (3.14) then gives the global error scheme

$$
\begin{aligned}
\varepsilon_{n+1 / 2}^{u} & =\varepsilon_{n}^{u}-\frac{1}{2} \tau K \varepsilon_{n}^{v}+\tau \delta_{1}, \\
\varepsilon_{n+1}^{v} & =\varepsilon_{n}^{v}+\tau K_{0}^{T} \varepsilon_{n+1 / 2}^{u}+\frac{1}{2} \tau K_{1}^{T}\left(\varepsilon_{n}^{u}+\varepsilon_{n+1}^{u}\right)-\frac{1}{2} \tau D\left(\varepsilon_{n}^{v}+\varepsilon_{n+1}^{v}\right)+\tau \delta_{2}, \\
\varepsilon_{n+1}^{u} & =\varepsilon_{n+1 / 2}^{u}-\frac{1}{2} \tau K \varepsilon_{n+1}^{v}+\tau \delta_{3} .
\end{aligned}
$$

Eliminating the intermediate error in the same symmetric way as in the derivation of the conservation relation yields

$$
\begin{aligned}
\varepsilon_{n+1}^{u}= & \varepsilon_{n}^{u}-\frac{1}{2} \tau K\left(\varepsilon_{n}^{v}+\varepsilon_{n+1}^{v}\right)+\tau \delta_{n}^{u}, \\
\varepsilon_{n+1}^{v}= & \varepsilon_{n}^{v}+\frac{1}{2} \tau K^{T}\left(\varepsilon_{n}^{u}+\varepsilon_{n+1}^{u}\right)-\frac{1}{2} \tau D\left(\varepsilon_{n}^{v}+\varepsilon_{n+1}^{v}\right) \\
& +\frac{1}{4} \tau^{2} K_{0}^{T} K\left(\varepsilon_{n+1}^{v}-\varepsilon_{n}^{v}\right)+\tau \delta_{n}^{v},
\end{aligned}
$$

where

$$
\begin{aligned}
& \delta_{n}^{u}=\delta_{1}+\delta_{3}, \\
& \delta_{n}^{v}=\delta_{4}+K_{0}^{T}\left(\frac{1}{2} \tau\left(\delta_{1}-\delta_{3}\right)+\delta_{5}\right) .
\end{aligned}
$$

These two new defects contain only even terms in $\tau$ and both start of with $\tau^{2}$. The defect $\delta_{n}^{u}$ is just the defect of the trapezoidal rule. At this stage we assume $u_{h}, v_{h} \in$ $C^{3}[0, T]$. Then it follows from the remainder in Taylor's theorem that

$$
\delta_{n}^{u}=\mathcal{O}\left(\tau^{2}\right), \quad \delta_{4}=\mathcal{O}\left(\tau^{2}\right), \quad \frac{1}{2} \tau\left(\delta_{1}-\delta_{3}\right)+\delta_{5}=\mathcal{O}\left(\tau^{2}\right) \quad \text { for } \tau \sim h, h \rightarrow 0 .
$$

Writing

$$
\begin{aligned}
& \left(\begin{array}{cc}
I & \frac{1}{2} \tau K \\
-\frac{1}{2} \tau K^{T} & I-\frac{1}{4} \tau^{2} K_{0}^{T} K+\frac{1}{2} \tau D
\end{array}\right)\left(\begin{array}{c}
\varepsilon_{n+1}^{u} \\
\varepsilon_{n+1}^{v}
\end{array}\right) \\
& =\left(\begin{array}{cc}
I & -\frac{1}{2} \tau K \\
\frac{1}{2} \tau K^{T} & I-\frac{1}{4} \tau^{2} K_{0}^{T} K-\frac{1}{2} \tau D
\end{array}\right)\left(\begin{array}{c}
\varepsilon_{n}^{u} \\
\varepsilon_{n}^{v}
\end{array}\right)+\tau\left(\begin{array}{c}
\delta_{u}^{n} \\
\delta_{v}^{n}
\end{array}\right),
\end{aligned}
$$


and putting $\varepsilon_{n}=\left[\left(\varepsilon_{n}^{u}\right)^{T},\left(\varepsilon_{n}^{v}\right)^{T}\right]^{T}$ and $\delta_{n}=\left[\left(\delta_{n}^{u}\right)^{T},\left(\delta_{n}^{v}\right)^{T}\right]^{T}$, we arrive at the compact notation

$$
\varepsilon_{n+1}=R \varepsilon_{n}+\tau \rho_{n}, \quad R=R_{L}^{-1} R_{R}, \rho_{n}=R_{L}^{-1} \delta_{n},
$$

with $R_{L}$ and $R_{R}$ the left and right block matrix, respectively.

This recursion has the standard form featuring in the convergence analysis of onestep integration methods, see e.g. [6]. Assuming Lax-Richtmyer stability, whereby we include $R_{L}$ inversely bounded for $\tau \sim h, h \rightarrow 0$, it transfers local errors to the global error by essentially adding all local errors. It trivially reveals second-order convergence for a fixed spatial dimension (ODE convergence) since then $K_{0}^{T}$ within the defect $\delta_{n}^{v}$ is bounded and hence $\rho_{n}=\mathcal{O}\left(\tau^{2}\right)$ for $\tau \rightarrow 0$, because both $\delta_{n}^{u}$ and $\delta_{n}^{v}$ are $\mathcal{O}\left(\tau^{2}\right)$. However, if we simultaneously refine the spatial grid, parameterized by $h \rightarrow 0$, the local error component $\delta_{n}^{v}$ must have components which will grow with $1 / h$. This growth is unavoidable, since by definition of $K_{1}=S_{u} K$ we have

$$
K_{0}^{T}=\left(K-K_{1}\right)^{T}=\left(\left(I-S_{u}\right) K\right)^{T}=K^{T}\left(I-S_{u}\right),
$$

showing that $K_{0}^{T}$ has zero columns and thus due to the splitting at certain components spatial consistency within the expression for $\delta_{n}^{v}$ is lost. This causes the growth by $1 / h$ which in turn causes reduction by one unit of $\tau$ if we let $\tau \sim h, h \rightarrow 0$ (order reduction). The local error component $\delta_{n}^{u}$ causes no problems. It is bounded for $h \rightarrow 0$ as it contains only solution derivatives.

Fortunately, in our case this order reduction by one unit of $\tau$ manifests itself only in the local error and cancels in the transition from local to global errors. That this cancelation occurs can be proven by transforming the global error scheme (3.22) into one with local errors which remain second order for $\tau \sim h, h \rightarrow 0$.

\subsubsection{A transformed global error recursion}

The following derivation, which we also used in [13] and which is based on a Lemma from [6], reveals that the second order will be maintained for any stable space-time grid refinement $\tau \sim h, h \rightarrow 0$. This derivation starts with the following Ansatz: the local error $\tau \rho_{n}$ allows a decomposition

$$
\tau \rho_{n}=(I-R) \xi_{n}+\eta_{n} \quad \text { such that } \xi_{n}=\mathcal{O}\left(\tau^{2}\right), \eta_{n}=\mathcal{O}\left(\tau^{3}\right)
$$

with order constants being $\mathcal{O}(1)$ for $\tau \sim h, h \rightarrow 0$.

Denote $\tilde{\varepsilon}_{n}=\varepsilon_{n}-\xi_{n}$. Then, by the Ansatz,

$$
\tilde{\varepsilon}_{n+1}=R \tilde{\varepsilon}_{n}+\tilde{\rho}_{n}, \quad \tilde{\rho}_{n}=\xi_{n}-\xi_{n+1}+\eta_{n}=\mathcal{O}\left(\tau^{3}\right),
$$

giving order two for $\tilde{\varepsilon}_{n}$ and hence also for $\varepsilon_{n}$. So there remains to check the Ansatz, which amounts to examining

$$
\tau \rho_{n}=\tau R_{L}^{-1} \delta_{n}=(I-R) \xi_{n}+\eta_{n}=\left(I-R_{L}^{-1} R_{R}\right) \xi_{n}+\eta_{n},
$$

or, equivalently,

$$
\tau R_{L}^{-1}\left(\begin{array}{c}
\delta_{n}^{u} \\
\delta_{n}^{v}
\end{array}\right)=R_{L}^{-1}\left(R_{L}-R_{R}\right)\left(\begin{array}{c}
\xi_{n}^{u} \\
\xi_{n}^{v}
\end{array}\right)+R_{L}^{-1} R_{L}\left(\begin{array}{c}
\eta_{n}^{u} \\
\eta_{n}^{v}
\end{array}\right),
$$


or

$$
\left(\begin{array}{c}
\tau \delta_{n}^{u} \\
\tau \delta_{n}^{v}
\end{array}\right)=\left(R_{L}-R_{R}\right)\left(\begin{array}{c}
\xi_{n}^{u} \\
\xi_{n}^{v}
\end{array}\right)+R_{L}\left(\begin{array}{c}
\eta_{n}^{u} \\
\eta_{n}^{v}
\end{array}\right)
$$

or

$$
\begin{aligned}
\tau\left(\delta_{1}+\delta_{3}\right)= & \tau K \xi_{n}^{v}+\eta_{n}^{u}+\frac{1}{2} \tau K \eta_{n}^{v}, \\
\tau\left(\delta_{4}+K_{0}^{T}\left(\frac{1}{2} \tau\left(\delta_{1}-\delta_{3}\right)+\delta_{5}\right)\right)= & -\tau K^{T} \xi_{n}^{u}-\frac{1}{2} \tau K^{T} \eta_{n}^{u}+\eta_{n}^{v} \\
& -\frac{1}{4} \tau^{2} K_{0}^{T} K \eta_{n}^{v}+\tau D\left(\xi_{n}^{v}+\frac{1}{2} \eta_{n}^{v}\right) .
\end{aligned}
$$

Thus, our task is now to identify error vectors $\xi_{n}^{u}, \xi_{n}^{v}$ and $\eta_{n}^{u}, \eta_{n}^{v}$ in accordance with the Ansatz such that (3.29) are satisfied. Let us first define

$$
\eta_{n}^{u}=\tau\left(\delta_{1}+\delta_{3}\right)=\mathcal{O}\left(\tau^{3}\right), \quad \xi_{n}^{v}=-\frac{1}{2} \eta_{n}^{v},
$$

where $\eta_{n}^{u}=\mathcal{O}\left(\tau^{3}\right)$ is due to (3.20). Then the first equation of (3.29) is satisfied in accordance with the Ansatz. Next we choose, again in accordance with the Ansatz,

$$
\eta_{n}^{v}=\tau \delta_{4}=\mathcal{O}\left(\tau^{3}\right)
$$

which is also due to (3.20). Then we are done if we can choose $\xi_{n}^{u}$ to satisfy

$$
K^{T} \xi_{n}^{u}=-\frac{1}{2} K^{T} \eta_{n}^{u}-\frac{1}{4} \tau K_{0}^{T} K \eta_{n}^{v}-K_{0}^{T}\left(\delta_{5}+\frac{1}{2} \tau\left(\delta_{1}-\delta_{3}\right)\right)
$$

such that $\xi_{n}^{u}=\mathcal{O}\left(\tau^{2}\right)$. Inserting $K_{0}^{T}=K^{T}\left(I-S_{u}\right)$ shows that

$$
\xi_{n}^{u}=-\frac{1}{2} \eta_{n}^{u}-\frac{1}{4} \tau\left(I-S_{u}\right) K \eta_{n}^{v}-\left(I-S_{u}\right)\left(\delta_{5}+\frac{1}{2} \tau\left(\delta_{1}-\delta_{3}\right)\right)
$$

satisfies (3.32). Thus there remains to confirm $\xi_{n}^{u}=\mathcal{O}\left(\tau^{2}\right)$. The first term of $\xi_{n}^{u}$ is $\mathcal{O}\left(\tau^{3}\right)$. Due to (1.3) we have $\tau K=\mathcal{O}(1)$ if $\tau \sim h, h \rightarrow 0$. Consequently, also the second term of $\xi_{n}^{u}$ is $\mathcal{O}\left(\tau^{3}\right)$. Finally, again using (3.20) shows that the third term is $\mathcal{O}\left(\tau^{2}\right)$, confirming that $\xi_{n}^{u}=\mathcal{O}\left(\tau^{2}\right)$. This completes the error analysis.

\section{Numerical results}

\subsection{Results in 1D}

To illustrate the technique of component splitting and the above second-order convergence result, we now present numerical tests for the one-dimensional Maxwell 
(wave) equation

$$
\begin{aligned}
& \frac{\partial H^{z}}{\partial t}=-\frac{\partial E^{y}}{\partial x}, \\
& \frac{\partial E^{y}}{\partial t}=-\frac{\partial H^{z}}{\partial x}-s(x, t),
\end{aligned}
$$

with a source $s(x, t)$ for component $E^{y}$. The tests for this one-dimensional Maxwell problem are academic but serve our purpose very well, as they illustrate the essential ideas put forward in this paper.

\subsubsection{Spatial discretization}

Assuming $0 \leq x \leq 1$, we introduce the grid $0=x_{0}<x_{1}<\cdots<x_{m-1}<x_{m}=$ 1 and discretize using standard staggering with $\tilde{v}_{i}(t) \approx E^{y}\left(x_{i}, t\right)$ and $\tilde{u}_{i}(t) \approx$ $H^{z}\left(x_{i-1 / 2}, t\right), x_{i-1 / 2}=\left(x_{i-1}+x_{i}\right) / 2$ (the tildes will be removed):

$$
\begin{aligned}
& \tilde{u}_{i}^{\prime}=-\frac{\tilde{v}_{i}-\tilde{v}_{i-1}}{x_{i}-x_{i-1}}, \quad i=1(1) m, \\
& \tilde{v}_{i}^{\prime}=-\frac{\tilde{u}_{i+1}-\tilde{u}_{i}}{x_{i+1 / 2}-x_{i-1 / 2}}-\frac{1}{x_{i+1 / 2}-x_{i-1 / 2}} \int_{x_{i-1 / 2}}^{x_{i+1 / 2}} s(x, t) d x, \quad i=1(1) m-1 .
\end{aligned}
$$

Herein $\tilde{v}_{0}$ and $\tilde{v}_{m}$ are located at the boundary and thus may stand for given boundary values. We assume this in the description below. For smooth solutions this approximation yields second-order spatial convergence on nonuniform grids, see e.g. [8].

To write this semi-discrete system in our generic form (1.4) we need to transform it. Let the grid functions $\tilde{u} \in \mathbb{R}^{m}$ and $\tilde{v} \in \mathbb{R}^{m-1}$ contain the components $\tilde{u}_{i}$ and $\tilde{v}_{i}$, respectively. Let the difference matrix $\tilde{K} \in \mathbb{R}^{m \times m-1}$ have entries $\tilde{K}_{i i}=1, i=$ $1(1) m-1$, and $\tilde{K}_{i+1 i}=-1, i=2(1) m$, and zeros elsewhere. Let $M_{d} \in \mathbb{R}^{m \times m}$ be diagonal with entries $\left(x_{i}-x_{i-1}\right)^{-1}$. Likewise, let $M_{h} \in \mathbb{R}^{m-1 \times m-1}$ be diagonal with entries $\left(x_{i+1 / 2}-x_{i-1 / 2}\right)^{-1}$. Then (4.2) can be written as

$$
\left(\begin{array}{l}
u^{\prime} \\
v^{\prime}
\end{array}\right)=\left(\begin{array}{cc}
0 & -K \\
K^{T} & 0
\end{array}\right)\left(\begin{array}{l}
u \\
v
\end{array}\right)+\left(\begin{array}{l}
f^{u}(t) \\
f^{v}(t)
\end{array}\right),
$$

where

$$
u=M_{d}^{-\frac{1}{2}} \tilde{u}, \quad v=M_{h}^{-\frac{1}{2}} \tilde{v}, \quad K=M_{d}^{\frac{1}{2}} \tilde{K} M_{h}^{\frac{1}{2}},
$$

and

$$
f^{u}(t)=M_{d}^{-\frac{1}{2}} \tilde{f}^{u}(t), \quad f^{v}(t)=M_{h}^{-\frac{1}{2}} \tilde{f}^{v}(t) .
$$

Herein $\tilde{f}^{u}(t) \in \mathbb{R}^{m}$ contains the boundary values, that is,

$$
\tilde{f}^{u}(t)=\left[-E^{y}\left(x_{0}, t\right) /\left(x_{1}-x_{0}\right), 0, \ldots, 0, E^{y}\left(x_{m}, t\right) /\left(x_{m}-x_{m-1}\right)\right]^{T},
$$

and, likewise, $\tilde{f}^{v}(t) \in \mathbb{R}^{m-1}$ contains the source values from (4.2) evaluated at the grid points $x_{i}$. System (4.3) is of the generic form (1.4) to be used for the numerical tests. 


\subsubsection{Test results}

We choose $0 \leq t \leq 1$ as time interval, a zero source function, impose the smooth exact solution $H^{z}(x, t)=\sin (\omega(x-t)), E^{y}(x, t)=H^{z}(x, t), \omega=2 \pi$, and compute this solution on the space grid depicted below. The grid is locally refined with a factor of one hundred around the center point $x=\frac{1}{2}$ which is also a grid point. In particular, we will keep the number of refined cells to the fixed amount of four for any coarse grid size $h$.

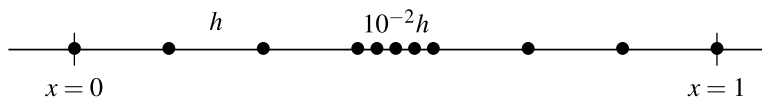

With this particular grid we thus aim to simulate a practical situation where solely due to spatial geometry a few very fine grid cells are needed [3], giving grid-induced stiffness. Needless to repeat that this is detrimental to the explicit integrator (2.1), as it severely limits the step size $\tau$ for stability. For our model problem and a uniform grid with grid size $h$, the critical step size for (2.1) is given by $\tau=h$ in accordance with (2.2). Due to the refining factor $10^{2}$, the critical step size for the depicted grid is expected to be close to $\tau=10^{-2} h$. It appears to be slightly larger, being $\tau \approx$ $1.08 \times 10^{-2} h$.

Next we illustrate the virtue of component splitting by comparing (2.1) applied with $\tau=10^{-2} h$ to the implicit trapezoidal rule (2.5) and the component splitting scheme (3.1), the latter two both applied with the one hundred times larger step size $\tau=h$. For (3.1) we still have to define the $m \times m$ diagonal splitting matrix $S_{u}$ introduced in (3.6). We have chosen

$$
S_{u}=\operatorname{diag}(0,0, \ldots, 0,0,1,1,1,1,1,0,0, \ldots, 0,0),
$$

that is, we have put all entries of $S_{u}$ equal to zero, except those five entries which belong to the components of $u$ that are connected with the fine grid cells centered around $x=\frac{1}{2}$. Those five are put equal to one, meaning that only fine grid cell information enters the matrix in (3.8) that features in the implicit solve. In other words, to overcome the stability limitation, with the component splitting scheme (3.1) we time step on the whole of the grid explicitly, except for the few fine cells. This is the essence of component splitting. In this simple 1D case, the dimension of the tridiagonal matrix $K_{1}^{T} K_{1}$ present in (3.8) is just six, whereas the dimension of $K^{T} K$ is equal to $m-2$. Needless to say that in 2D or 3D such savings are appealing.

Figure 1 plots for increasing numbers of grid cells the maximum of all absolute space-time errors of $H^{z}, E^{y}$ at time $t=1$. The plots clearly confirm the second-order convergence result of the component splitting scheme for $h \rightarrow 0$ and, in this case, show in addition favorable space-time errors for this scheme compared to the explicit and implicit one. Further tests with different component splittings also confirmed the second-order convergence result. 
Fig. 1 Convergence plots for 1D test: o-marker component splitting, *-marker explicit, +-marker implicit. The dashed line has slope two for second-order convergence

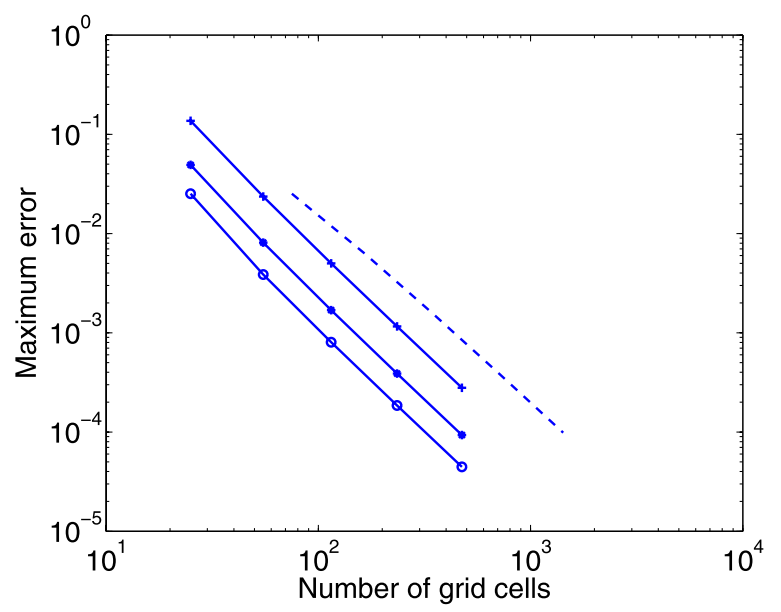

\subsection{Results in $2 \mathrm{D}$}

For a 2D test we consider the TM (transversal magnetic) model with components $H^{x}, H^{z}$ and $E^{y}$,

$$
\begin{aligned}
\mu \frac{\partial H^{x}}{\partial t} & =\frac{\partial E^{y}}{\partial z}, \\
\mu \frac{\partial H^{z}}{\partial t} & =-\frac{\partial E^{y}}{\partial x}, \\
\frac{\partial E^{y}}{\partial t} & =\frac{\partial H^{x}}{\partial z}-\frac{\partial H^{z}}{\partial x} .
\end{aligned}
$$

For this model we borrow a test setup from [13] based on a strongly varying function $\mu(x, z)$ rather than using a locally refined grid. This test setup is also academic, but like for the $1 \mathrm{D}$ problem it nicely illustrates the ideas behind the technique of component splitting. The function $\mu(x, z)$ is chosen as the inverse of the peaked function

$$
d(x, z)=1.0+\left(d_{m}-1.0\right) e^{\left.-d_{s}\left((x-0.5)^{2}+(z-0.5)^{2}\right)\right)} .
$$

With $d_{s}>0$ we can monitor the shape and with $d_{m}>1$ the height. The larger $d_{m}$, the greater the step size restriction for the explicit method. Thus the idea of the test is to take $d$ close to one almost everywhere and to use component splitting near a high and local peak so as to avoid the step size restriction over the whole space domain.

For the space domain we take the unit square and impose component $E^{y}$ zero at the boundary (boundary conditions for $H^{x}$ and $H^{z}$ are not needed). We choose as initial conditions at time $t=0$ the zero function for $H^{x}$ and $H^{z}$ and let $E^{y}(x, z, 0)=$ $\sin (\beta x) \sin (\beta z), \beta=2 \pi$. The resulting exact solution is not available in analytical form. Therefore, for assessing our test results, we will use a sufficiently accurate reference (semi-discrete) solution on the chosen space grids. 


\subsubsection{Spatial discretization}

We semi-discretize (4.8) by means of second-order, central differences on a uniform, staggered grid with grid size $h=1 / m$. Let $x_{i}=i h, x_{i+1 / 2}=(i+1 / 2) h$, etc. Then, $E^{y}$ is approximated at $\left(x_{i}, z_{j}\right)$ for $i, j=1(1) m-1, H^{x}$ at $\left(x_{i}, z_{j+1 / 2}\right)$ for $i=1(1) m-1$ and $j=0(1) m-1$, and $H^{z}$ at $\left(x_{i+1 / 2}, z_{j}\right)$ for $i=0(1) m-1$ and $j=1(1) m-1$. Note that this staggering accommodates our boundary condition because due to the staggering $H^{x}$ and $H^{z}$ are not required at the domain boundary.

Assuming natural ordering along grid lines in the $x$-direction, let $\tilde{u}_{1} \in \mathbb{R}^{m(m-1)}$ denote the (grid function) vector for $H^{x}$ resulting from the staggered discretization (the tildes will be removed). Similarly, let $\tilde{u}_{2} \in \mathbb{R}^{m(m-1)}$ denote this vector for $H^{z}$ and $\tilde{v} \in \mathbb{R}^{(m-1)(m-1)}$ for $E^{y}$. The resulting semi-discrete system then takes the form

$$
\left(\begin{array}{c}
M_{1} \tilde{u}_{1}^{\prime} \\
M_{2} \tilde{u}_{2}^{\prime} \\
\tilde{v}^{\prime}
\end{array}\right)=\left(\begin{array}{ccc}
0 & 0 & \tilde{E}_{[z]}^{y} \\
0 & 0 & -\tilde{E}_{[x]}^{y} \\
\tilde{H}_{[z]}^{x} & -\tilde{H}_{[x]}^{z} & -\tilde{D}
\end{array}\right)\left(\begin{array}{c}
\tilde{u}_{1} \\
\tilde{u}_{2} \\
\tilde{v}
\end{array}\right),
$$

where $M_{1}, M_{2}$ and $\tilde{D}$ are diagonal matrices containing the encountered $\mu$-values and $\sigma$-values (taken unequal zero for the time being), respectively. Further, $\tilde{E}_{[z]}^{y}$ is the difference matrix for $\partial / \partial z$ acting on component $E^{y}$, etc. For the staggered grid holds

$$
\tilde{H}_{[z]}^{x}=-\left(\tilde{E}_{[z]}^{y}\right)^{T}, \quad \tilde{H}_{[x]}^{z}=-\left(\tilde{E}_{[x]}^{y}\right)^{T},
$$

giving

$$
\left(\begin{array}{c}
M_{1} \tilde{u}_{1}^{\prime} \\
M_{2} \tilde{u}_{2}^{\prime} \\
\tilde{v}^{\prime}
\end{array}\right)=\left(\begin{array}{ccc}
0 & 0 & \tilde{E}_{[z]}^{y} \\
0 & 0 & -\tilde{E}_{[x]}^{y} \\
-\left(\tilde{E}_{[z]}^{y}\right)^{T} & \left(\tilde{E}_{[x]}^{y}\right)^{T} & -\tilde{D}
\end{array}\right)\left(\begin{array}{c}
\tilde{u}_{1} \\
\tilde{u}_{2} \\
\tilde{v}
\end{array}\right) .
$$

This form reveals the skew-symmetry for zero $\tilde{D}$.

Finally, to arrive at our generic form (1.4), we rescale (4.12) to

$$
\left(\begin{array}{c}
u_{1}^{\prime} \\
u_{2}^{\prime} \\
v^{\prime}
\end{array}\right)=\left(\begin{array}{ccc}
0 & 0 & E_{[z]}^{y} \\
0 & 0 & -E_{[x]}^{y} \\
-\left(E_{[z]}^{y}\right)^{T} & \left(E_{[x]}^{y}\right)^{T} & -D
\end{array}\right)\left(\begin{array}{c}
u_{1} \\
u_{2} \\
v
\end{array}\right),
$$

where

$$
u_{1}=M_{1}^{1 / 2} \tilde{u}_{1}, \quad u_{2}=M_{2}^{1 / 2} \tilde{u}_{2}, \quad v=\tilde{v},
$$

and

$$
E_{[z]}^{y}=M_{1}^{-1 / 2} \tilde{E}_{[z]}^{y}, \quad E_{[x]}^{y}=M_{2}^{-1 / 2} \tilde{E}_{[x]}^{y}, \quad D=\tilde{D} .
$$


It follows that the matrix $K$ from $(1.4)$ is given by the $2 m(m-1) \times(m-1)^{2}$ difference matrix

$$
K=\left(\begin{array}{c}
-E_{[z]}^{y} \\
E_{[x]}^{y}
\end{array}\right)=\left(\begin{array}{c}
-M_{1}^{-1 / 2} \tilde{E}_{[z]}^{y} \\
M_{2}^{-1 / 2} \tilde{E}_{[x]}^{y}
\end{array}\right) .
$$

Clearly, matrix $S_{u}$ introduced in (3.6) and (3.7) thus takes the form

$$
S_{u}=\left(\begin{array}{c}
S_{u_{1}} \\
S_{u_{2}}
\end{array}\right)
$$

where the diagonal matrices $S_{u_{1}}$ and $S_{u_{2}}$ multiply $-E_{[z]}^{y}$ and $E_{[x]}^{y}$, respectively. These diagonal matrices define the splitting. They will be given below.

\subsubsection{Test results}

We choose for (4.9) the coefficient values $d_{m}=100, d_{s}=2000$ which gives a strongly peaked shape with a height of one hundred. Test runs are carried out on five space-time grids defined by $h=1 / 20,1 / 40,1 / 80,1 / 160,1 / 320$ and $\tau=\tau(h)$ given by the corresponding stability step size restriction for the explicit scheme away from the peak where $d$ is close to one.

We realize this as follows. For a spatially constant coefficient function $d$, the stability step size restriction for the explicit scheme is

$$
\tau \leq \tau_{c}=\frac{h}{\sqrt{2 d}}
$$

Without splitting this would result in a step size restriction $\tau \leq h / \sqrt{200}$. The idea is now to choose the entries of the splitting matrices $S_{u_{1}}$ and $S_{u_{2}}$ introduced in (4.17) equal to one if the corresponding entries in the matrices $M_{1}$ and $M_{2}$ of system (4.10) are $\leq \frac{1}{2}$, that means, the coefficient values $d(x, z) \geq 2$. Otherwise the entries of $S_{u_{1}}$ and $S_{u_{2}}$ are taken zero. Hence we use implicit time stepping only in the vicinity of the peak, and elsewhere explicit time stepping. The threshold $d(x, z) \geq 2$ suggests that, in accordance with (4.18), the required step size for the splitting scheme is

$$
\tau=\frac{h}{\sqrt{2 d}} \quad \text { with } d=2 .
$$

Heuristically, we then satisfy the stability step size condition everywhere, while saving a factor $\sqrt{50}$ for the step size, of course at the expense of some implicit computations.

Results for the splitting scheme (3.1) and the fully implicit one (2.5), obtained with the same step sizes $\tau$, are listed in Table 1, including maximum errors taken over all components at time $t=1$ and numbers nnz (numbers of nonzeros) of the matrices $K_{1}^{T} K_{1}$ for the splitting scheme and $K^{T} K$ for the implicit scheme. These numbers measure the sparsity encountered in the solution of the linear system (3.4). Note that in this 2D example, the matrix $K^{T} K$ is the five-banded, second-order difference matrix for the second-order elliptic operator. Matrix $K_{1}^{T} K_{1}$ is similar, but contains 
Table 1 Maximum norm errors and nnz's for the 2D test problem

\begin{tabular}{rlrlr}
\hline$m$ & scheme (2.5) & \multicolumn{1}{c}{ nnz } & scheme (3.1) & nnz \\
\hline 20 & $0.20910^{-1}$ & 1729 & $0.12310^{-1}$ & 13 \\
40 & $0.27010^{-1}$ & 7449 & $0.26810^{-1}$ & 69 \\
80 & $0.18610^{-1}$ & 30889 & $0.12710^{-1}$ & 233 \\
160 & $0.72410^{-2}$ & 125769 & $0.72010^{-2}$ & 965 \\
320 & $0.53710^{-2}$ & 507529 & $0.43110^{-2}$ & 3753 \\
\hline
\end{tabular}

only the rows and columns of $K^{T} K$ near the peak in accordance with the chosen threshold for $d(x, z) \geq 2$. All other rows and columns of $K_{1}^{T} K_{1}$ are zero.

As can be seen in Table 1, the component splitting scheme yields the same errors as the implicit trapezoidal rule. The fact that the second order does not show up on the chosen space-time grids, is due to the very large gradients of the coefficient function (4.9). These necessarily enter the discretization error. Taking the peak more smooth will reveal second order. Furthermore, without a loss of accuracy we see that for the component splitting scheme the numbers nnz are significantly lower as anticipated. This, of course, is what we have forced here by the threshold $d(x, z) \geq 2$. Yet it again illustrates the virtue of component splitting, now in a setting different from that of the $1 \mathrm{D}$ test problem.

\section{Future work}

The practical virtue of the component splitting scheme (3.1) for real applications has not been illustrated in this paper and has to be assessed of course. In the near future we plan to carry out such an assessment for discontinuous Galerkin discretizations on unstructured meshes, as part of joint research with the authors from [3]. With unstructured meshes covering irregular domains, grid induced stiffness is likely to happen. Component splitting can overcome this at the expense of a small overhead for local implicit computations. This joint research will also deal with a comparison of our scheme with a related component splitting scheme from [3]. The scheme from [3] differs from (3.1) in that it was especially designed for discontinuous Galerkin discretizations on unstructured meshes.

Because with the discontinuous Galerkin approach it is possible to increase the spatial convergence order, within this joint research we also plan to assess the practical virtue of component splitting for higher-order integration schemes. One possibility is to examine higher-order compositions [5]. For (3.1) this amounts to

$$
\Psi_{\tau}=\Phi_{\alpha_{s} \tau} \circ \Phi_{\beta_{s} \tau}^{*} \circ \cdots \circ \Phi_{\alpha_{1} \tau} \circ \Phi_{\beta_{1} \tau}^{*},
$$

with $\Phi_{\tau}$ given by

$$
\begin{aligned}
& \frac{u_{n+1}-u_{n}}{\tau}=-K v_{n+1}+f^{u}\left(t_{n+1}\right), \\
& \frac{v_{n+1}-v_{n}}{\tau}=K_{0}^{T} u_{n}+K_{1}^{T} u_{n+1}-D v_{n+1}+f^{v}\left(t_{n+1}\right) .
\end{aligned}
$$


The composition $\Psi_{\tau}=\Phi_{\tau / 2} \circ \Phi_{\tau / 2}^{*}$ is identical to (3.1) and, for example, ODE order four can be achieved for $s=5$ with coefficients from [7]. Whether these higher-order extensions will maintain their ODE order for spatial grid size $h \rightarrow 0$ is doubtful. Yet they can be efficient because well-designed composition coefficients, such as those from [7], minimize truncation error coefficients to the extent that order reduction becomes less deficient.

As already mentioned in Sect. 1, the method from [2] and [4] also serves to overcome step size limitations by grid-induced stiffness. That method is designed for second-order wave equations and the work reported focuses also on the Maxwell equations. It is based on the two-step leapfrog method and overcomes the step size limitation by local time stepping which renders the method explicit. In that respect it differs substantially from the component splitting method from this paper and from the one from [3]. The base method is also second-order accurate and can handle conduction terms too. Higher order is achieved through the modified equation approach, but only for zero conduction. The second- and higher-order local time-stepping methods are no doubt very interesting. A numerical comparison between the local timestepping methods and the methods from this paper and [3] is of interest, certainly so if $3 \mathrm{D}$ problems close to the actual practice would be considered.

Acknowledgements Stéphane Descombes and Stéphane Lanteri are acknowledged for sharing ideas and experiences on component splitting and for developing plans for future co-operation.

\section{References}

1. Botchev, M.A., Verwer, J.G.: Numerical integration of damped Maxwell equations. SIAM J. Sci. Comput. 31, 1322-1346 (2009)

2. Diaz, J., Grote, M.J.: Energy conserving explicit local time stepping for second-order wave equations. SIAM J. Sci. Comput. 31, 1985-2014 (2009)

3. Dolean, V., Fahs, H., Fezoui, L., Lanteri, S.: Locally implicit discontinuous Galerkin method for time domain electromagnetics. J. Comput. Phys. 229, 512-526 (2010)

4. Grote, M.J., Mitkova, T.: Explicit local time stepping methods for Maxwell's equations. J. Comput. Appl. Math. 234, 3283-3302 (2010)

5. Hairer, E., Lubich, C., Wanner, G.: Geometric Numerical Integration, 2nd edn. Springer Series in Computational Mathematics, vol. 31, Springer, Berlin (2006)

6. Hundsdorfer, W., Verwer, J.G.: Numerical Solution of Time-Dependent Advection-DiffusionReaction Equations. Springer Series in Computational Mathematics, vol. 33. Springer, Berlin (2003)

7. McLachlan, R.I.: On the numerical integration of ordinary differential equations by symmetric composition methods. SIAM J. Sci. Comput. 16, 151-168 (1995)

8. Monk, P., Süli, S.: A convergence analysis of Yee's scheme on nonuniform grids. SIAM J. Numer. Anal. 31, 393-412 (1994)

9. Rodrigue, G., White, D.: A vector finite element time-domain method for solving Maxwell's equations on unstructured hexahedral grids. SIAM J. Sci. Comput. 23, 683-706 (2001)

10. Sanz-Serna, J.M., Verwer, J.G., Hundsdorfer, W.H.: Convergence and order reduction of Runge-Kutta schemes applied to evolutionary problems in partial differential equations. Numer. Math. 50, 405-418 (1987)

11. Verlet, L.: Computer "experiments" on classical fluids. I. Thermodynamical properties of LennartJones molecules. Phys. Rev. 159, 98-103 (1967)

12. Verwer, J.G., Botchev, M.A.: Unconditionally stable integration of Maxwell's equations. Linear Algebra Appl. 431, 300-317 (2009)

13. Verwer, J.G.: Convergence and component splitting for the Crank-Nicolson-Leap-Frog integration method. CWI Report MAS-E0902. http://ftp.cwi.nl/CWIreports/MAS/MAS-E0902.pdf (2009)

14. Yee, K.S.: Numerical solution of initial boundary value problems involving Maxwell's equations in isotropic media. IEEE Trans. Antennas Propag. 14, 302-307 (1966) 